\title{
GLOBULAR CLUSTERS AND PRIMORDIAL COMPOSITION
}

\author{
Roger Cayre1 \\ Observatory of Paris
}

\section{INTRODUCTION}

So much has been learned already, during these last four days, about globulars that I shall start by addressing the second part of my topic first: the primordial chemical composition. Then I shall review the abundance of the primordial elements in globulars, or more generally in Population II. Actually if the globulars are the oldest stars ever observed in the Universe one could hope that the subject is over and that there is no need to speak about elements synthesized in stellar interiors for globulars. Unfortunately these rascals show always some amount of stellar nucleosynthesis products and this may very well be the central problem raised by their chemical composition. We shall discuss in turn the amount of the stellar synthesized elements in globulars and then the clues given by the pecularities of the abundance ratios, which may help in understanding their origin. The last section summarized the present knowledge of the subject.

\section{THE PRIMORDIAL COMPOSITION}

Let us now turn our attention towards a refreshingly simple physical system (when compared to a globular cluster): the Universe as a whole.

Table I summarizes the main events which we believe to have occured in the Universe. The period of interest for the building up the primordial composition is between $0.1 \mathrm{~s}$ and $1000 \mathrm{~s}$. At the time $0.1 \mathrm{~s}$ the Universe was indeed a very simple system: almost perfect uniformity in temperature and density, interactions in the domain of $1 \mathrm{Mev}$ with $\mathrm{T} \simeq$ $10^{10} \mathrm{~K}$. No departure from LTE, no molecules, and every species extraordinarily close to thermodynamic equilibrium. One could think that the word 'local' does not sound right when one is speaking of the whole Universe but at this time the 'causal' horizon was encompassing a mass of

the order of $10^{-3} \mathrm{~m}_{\odot}$ only, so the nucleosynthesis has been more local than what could be thought a first view. The physical basis for the study of the elaboration of the primordial composition have already been

431

J. E. Grindlay and A. G. Davis Philip (eds.),

The Harlow-Shapley Symposium on Globular Cluster Systems in Galaxies, 431-442.

(C) 1988 by the IAU. 
TABLE I

Time-Table of the Big-Bang

time

(s)

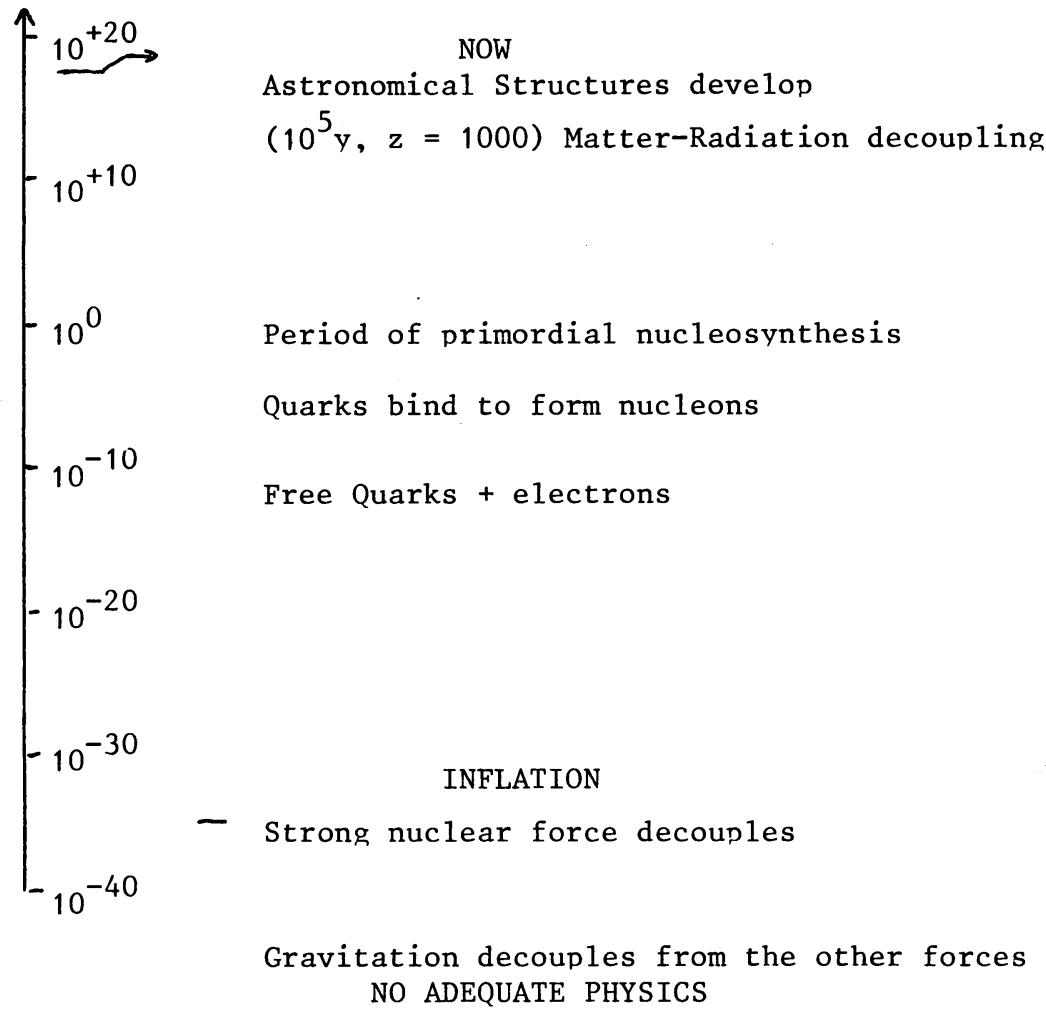

established by Wagoner 1973, but the basic recent paper on the subject is by Yang and a1. 1984, and a nice review of both the theoretical aspect and the observational evidence concerning the primordial elements is given by Boesgaard and Steigman 1985 .

What has driven some action in the physical conditions of the Universe between the time $0.1 \mathrm{~s}$ and $1000 \mathrm{~s}$ is of course the expansion. The semi-thermodvnamical equilibrium existing at the time $0.1 \mathrm{~s}$ has not been able to survive because of the cooling of the Universe and of the density decrease, both factors leading to a decrease of all the reaction rates down to practically zero at the time 1000s ('freeze out' of the nuclear composition). All the processes do not freeze out at the same time, because rates do not decline all equally. A close up of the main events in the period of primordial nucleosynthesis is given in table II. 
TABLE II

Important events during Nucleosynthesis period

\begin{tabular}{|c|c|}
\hline Time（s） & Event \\
\hline 0,1 & $\begin{array}{l}\text { neutrinos decouple, proton and neutron } \\
\text { in statistical equilibrium }\end{array}$ \\
\hline 1 & protons and neutrons decouple \\
\hline 10 & $\begin{array}{l}\mathrm{e}^{+}, \mathrm{e}^{-} \text {pair production stops } \\
\mathrm{N}(\text { nucleons)/N(photons) freezes out. } \\
\text { Deuterium production begins }\end{array}$ \\
\hline 30 & ${ }^{3} \mathrm{He}$ and ${ }^{4} \mathrm{He}$ production starts \\
\hline 300 & $\mathrm{Li}$ production starts \\
\hline 1000 & $\begin{array}{l}\text { all reaction rates become unsignificant, } \\
\text { Big-Bang primordial composition is set. }\end{array}$ \\
\hline
\end{tabular}

The predicted frozen composition does depend upon a single parameter $\eta$, the ratio between the number of nucleons and the number of photons (conserved later during the expansion until, of course, non primeval photons have been generated bu nuclear burning in stars). Fig.1 shows the predicted abundances as a function of $\eta$, under the usual assumptions that the metrics was of the Robertson-Walker type, and that the exDansion rate was controlled by the equations of General Relativity. To this, one must add that the number of Relativistic particles existing was known ( 3 mass-loss neutrinos are usually assumed). Within the frame of the Grand Unified Theory other particles may have existed (photinos, gravitinos, axions) and if this is the case their contribution to the density may have to be included, and the expansion rate would be affected. For a discussion of this point see Boesgaard and Steigman 1985 or Audouze 1986). We shall only be concerned today with the so called 'standard' Big-Bang for which these new particules are not taken into account.

The direct or (indirect) best evidence for the initial abundances of the primordial elements is shown in Fig.1. It is very very remarkable that they agree more or less, and are not orders of magnitude apart from each other. The most accurate evidence for the primordial abundance of $4 \mathrm{He}$ does not come from old stars but from the ${ }^{4} \mathrm{He} / \mathrm{H}$ ratio in extragalactic H II regions of low metallicity(Kunth and Sargent 1983, Peimbert 1986). The case of $3 \mathrm{He}$ and $\mathrm{D}$ is more involved as these elements have been subjected to some amount of astration which has to be estimated. 


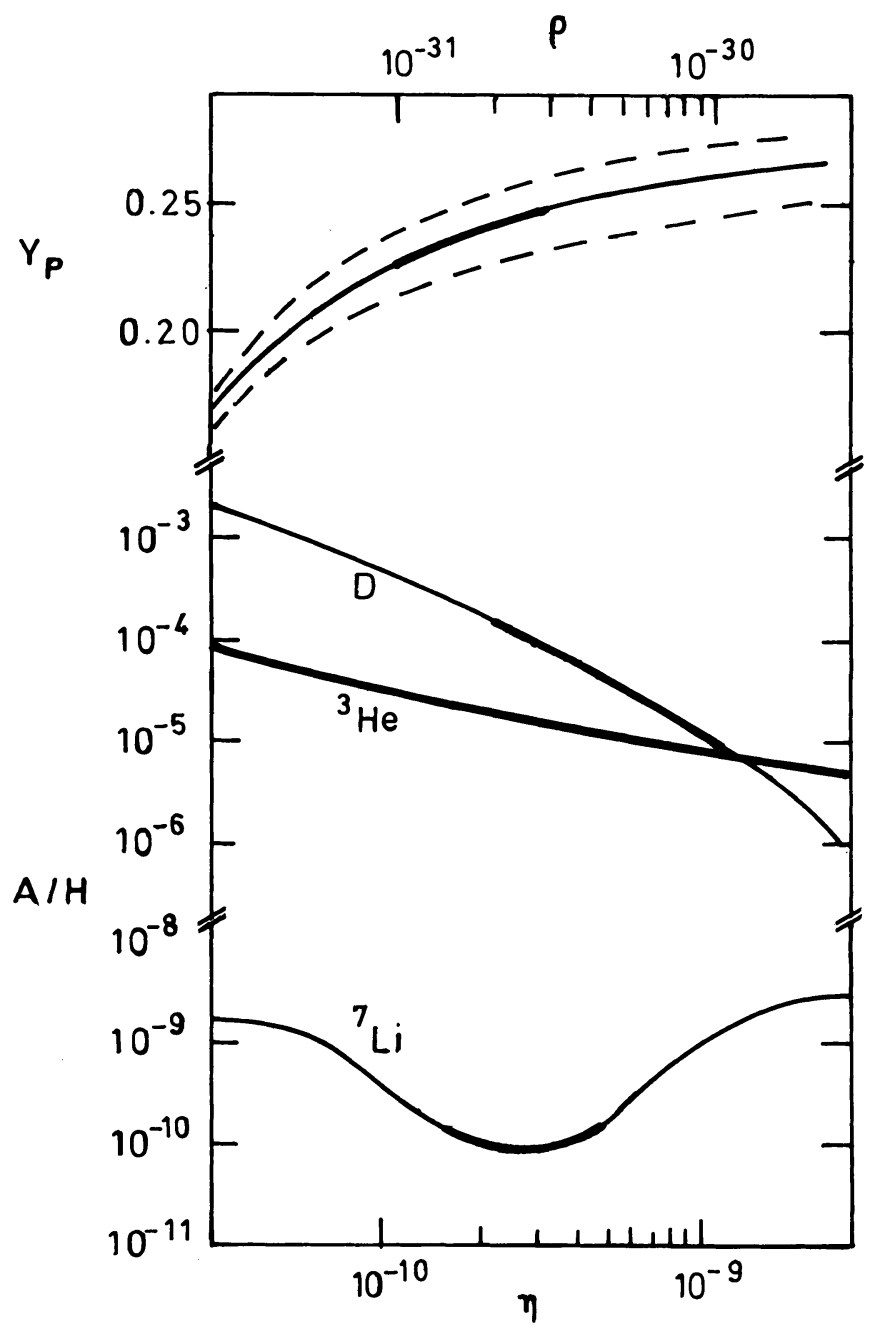

Fig. 1. Predicted primordial abundances in the 'standard' Big-Bang, according to Yang et al.. Note that in the upper part the ${ }^{4} \mathrm{He}$ abundance $\mathrm{Y}_{\mathrm{p}}$ is by mass, whereas for $\mathrm{D},{ }^{3} \mathrm{He}$ and ${ }^{7} \mathrm{Li}$ the abundances are by number of particles, relative to $\mathrm{H}$. The abscissa at the lower edge of the frame is the number ratio of nucleons to photons, whereas the abscissa at the upper edge is the present baryonic mean density of the Universe in $\mathrm{gcm}^{-3}$. On each curve the values compatible with observational evidence are reinforced. The three curves for $4 \mathrm{He}$ are respectively for a number of neutrons species equal to 2,3 and 4, from bottom to top. The assumed neutron life-time is 10.6 minutes. 


\section{THE PRIMORDIAL ELEMENTS IN GLOBULARS}

\subsection{Helium}

If the extrapolation of the helium abundance to zero $0 / \mathrm{H}$ ratio in oxygen-poor $\mathrm{H}$ II regions represents the primordial helium abundance how does this value compare to the helium abundance in globulars? This last one can be determined by several methods : the position of the blue edge of the instability trio in the color-luminosity diagram, the position of the main sequence in the theoretical HR diagram, the magnitude difference between the horizontal branch (HB) and a point of the unevo1ved main sequence of given effective temperature, the ratio of the number of stars on the $\mathrm{HB}$ and on the red giant branch (RB), etc... These methods have been reviewed recently by Caputo and Castellani (1983) and Cole et al. (1983). The most accurate method is probably the last one mentioned. Buzzoni et al. (1983) give:

$$
\mathrm{Y}_{\mathrm{GC}}=0.23 \pm 0.02
$$

in agreement, within the error bars, with the primordial abundance $\mathrm{Y}_{\mathrm{p}}$ :

$$
\mathrm{Y}_{\mathrm{p}}=0.24 \pm 0.01
$$

derived from the study of oxygen poor extragalactic H II regions (Kunth 1986). The accuracy of such determinations has been discussed recently (Davidson and Kinman 1985).

\subsection{Deuterium and $3_{\mathrm{He}}$}

These elements are not observable in low mass star Population II.

\subsection{Lithium}

By far this is the most interesting element. By an incredible luck, metal-poor stars have a much shallower convective zone than population I stars. Lithium, which is burnt in all Population I unevolved stars as old as the sun, has survived in Population II main sequence stars with metallicities lower than $1 / 20$ solar, and with an effective temperature larger than 5500 K. ( Spite 2 1982, Spite et a1. 1984, Spite2 1986). Globular cluster main-sequence stars are too faint to allow a good spectroscopic determination of lithium abundance but the parent population of field subdwarfs has been well studied by the Spites, who have found the results shown in Fig. 2 from a nearly complete sample of subdwarfs brighter than $V=9.5$. Lithium is the only element which does not show any correlation with $\mathrm{Fe} / \mathrm{H}$, and this constitutes the most direct evidence that this element is primordial. All other elements show a strong correlation with $\mathrm{Fe} / \mathrm{H}$, with a slope 1, when one does consider a pure halo star sample (François 1986). The only other element which has been claimed to show a slope significantly lower than 1 is nickel (Luck and Bond 1983), but further work by other authors has not confirmed this result. One should note that the abundance found for lithium in popu- 


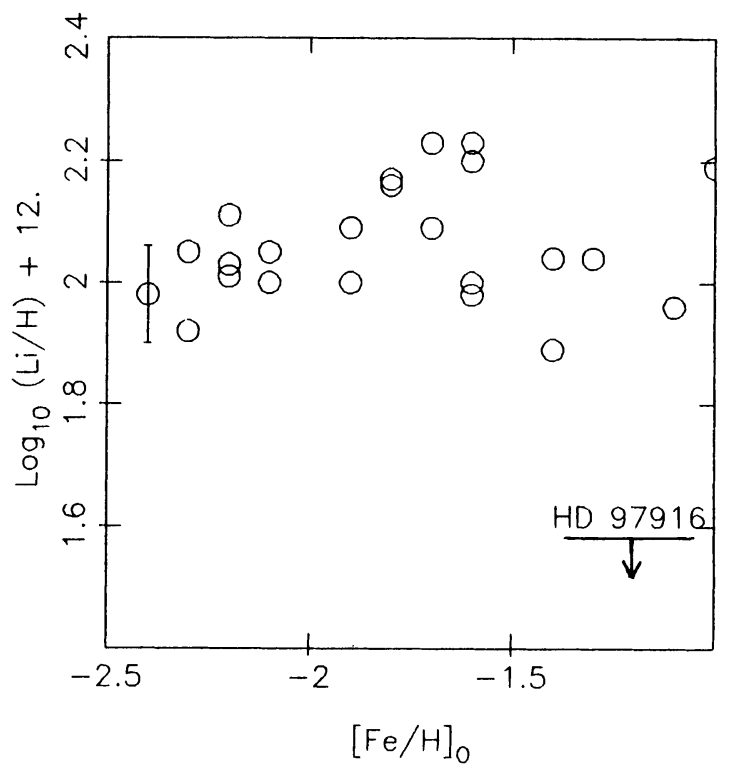

Fig. 2. Lithium abundance found by the Spites in subdwarfs as a function of $[\mathrm{Fe} / \mathrm{H}]$. Note the independence of the lithium abundance vs. $[\mathrm{Fe} / \mathrm{H}]$. This is a unique case among all other elements.

lation II subdwarfs is 10 times smaller than the so-called cosmic abundance $\left(\mathrm{Li} / \mathrm{H}=10^{-9}\right.$ ) found in young population $\mathrm{I}$ stars and in the today interstellar matter.

\section{THE METALLICITY OF GLOBULAR CLUSTERS.}

During a couple of decades the metallicity of globulars was thought to be the result of progressive enrichment of the Galaxy in elements synthesized in stellar interiors and rejected into the interste1lar medium, mostly by supernovae explosions (Eggen, Lynden, Be11, Sandage 1962).

However, if GC are the oldest objects in our Galaxy and in other galaxies as well, how is it that none of them has the primordial composition which was the only possibility prior to enrichment? This problem which was shown by Bond 1981, to occur as well in the field population II, is the so-called population III problem. Because we are more concerned in this symposium by GC than by field population II, and also because the metallicity distribution of field population II is still a matter of controversy (see Poster $n^{\circ} 108$ ), we shall discuss mostly the metallicity of globular clusters. Zinn 1985 has shown that the histogram of the metallicity of globular clusters has a double peak when the logarithm of the metallicity is used for abscissa. 
The metal rich group has kinematical properties resembling a thick disk population rather than a spheroidal population. If, in order to discuss the evolution of the metallicity according to current galaxy enrichment models we use the metallicity instead its logarithm, we found the results shown in Fig. 3 and 4 . On a coarse scale (Fig.3) the first peak appears as an intense 'flash' of production of heavy elements at the very beginning of the life of the Galaxy, whereas the second peak is smeared out and has the aspect of an 'on-going' activity at a much lower level. We believe that the progressive enrichment model of Eggen. Lynde1-Bell and Sandage does describe this second family of GC, but that the initial flash shown at an enlarged scale on Fig. 4 may have little to do with anything 'nrogressive'. An important sten was accomplished by Searle 1977 who postulated that GC were born of 'fragments' of interstellar matter orbiting freely in the galactic gravitational field prior to the formation of the disk. This assumption allows to understand the fact that the metallicity of GC appears to be independent of galactocentric distance, as shown already in this meeting. In fact the metallicity depends only on what has happened in each of these fragments. However this model keeps the progressive enrichment model in each of the fragments, and for this reason has the almost horizontal start inherent to the so-called simple model, for which the amount of heavy elements producted up to time $t$, is proportional to the number of low mass stars produced within the same laps of time. Let us note that a purely stochastic law (log-normal) fits as we11, and indeed better, the observed histogram.

So the basic question is to understand, why even if we extend our sample to extragalactic GC, there is no GC with metallicity significantly lower than $1 / 200$ of the solar metallicity. Two types of explanations have been proposed to explain this fact:

i) There was a pregalactic stellar generation, with an initial mass function truncated below $0.8 \mathrm{~m}$, which has produced heavy elements but no shining star, only invisible remnants.

ii) The star formation in a collapsing primordial cloud has suffered some amount of self-pollution, and low mass stars have been po1luted by type II Supernovae resulting of fast evolving 0 stars. Explanation 1) has been first advocated by Truran and Cameron 1971. A variant of it by Carr, Bond and Arnett 1984 proposes that the first (pregalactic' generation is made of very massive objects (VMOs), with masses in the range 100 to $1000 m_{\odot}$. The remnants of such objects would be of course invisible now.

The physical reason justifying a cut-off of the initial mass function below $0.8 \mathrm{~m} \odot$ in a zero metal environment is the absence of radiative mechanism otherwise available in present day interstellar matter. However when one includes $\mathrm{H}_{2}$ formation (Palla 1983) the Jeans mass may well be as small as $0.1 \mathrm{Tn}_{0}$ even in a metal free medium.

Explanation ii) has been originally proposed by Peebles and Dicke 1968. The existence of the large structures of the Universe makes unli- 


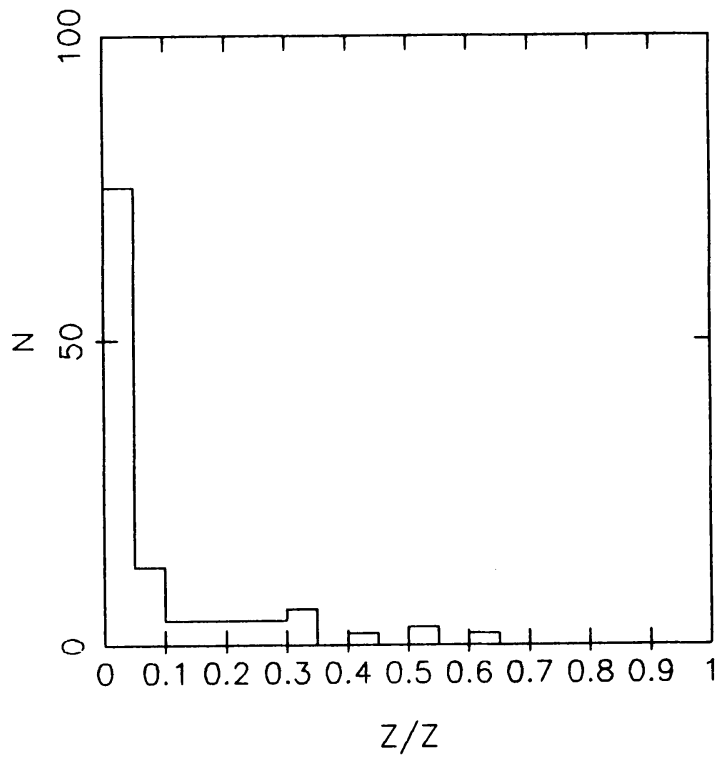

Fig. 3. The histogram of the metallicity of GC with bins on a linear scale in $Z$. (coarse scale). The source is Zinn 1985.

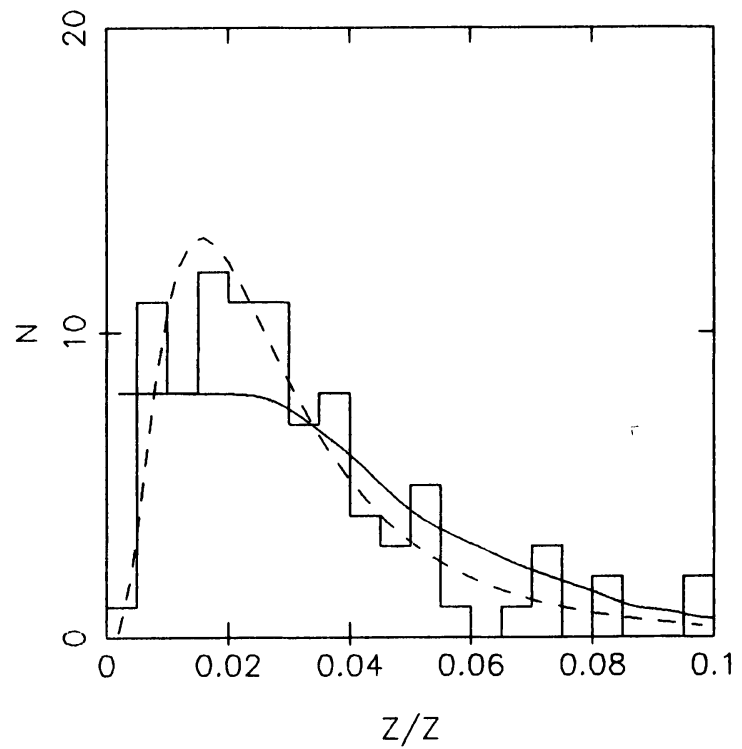

Fig. 4. The histogram of the metallicity of GC with bins on a linear scale in $\mathrm{Z}$ (finer scale, showing the low metallicity part). The full curve is according to Searle 1977, the dashed curve is a fitted lognormal law with $[\mathrm{Fe} / \mathrm{H}]=-1.6$ and dispersion $\sigma=0.3 \mathrm{dex}$. 
kely that GC did form first as in this 'bottom-up' cosmological model, but recently the author of this review has developped the idea that even if the earlv galaxy was made of orimordial clouds, the gravitational collapse of such clouds may have formed first a central dense nucleus rich in verv massive stars which. in becoming SN II. have po1luted the cloud itself before low mass stars had time to develop (Cayre1 1986).

At the present time there is no definite proof on which one of these two explanations holds more truth.

\section{ABUNDANCE RATIOS IN GLOBULAR CLUSTER STARS}

If it is well known that population II is metal poor, the question of how do differ the abundances ratios in population II and in population I has not been very clear until recent work. The recommended references on the subject are Spite ${ }^{2}$ 1985, and François 1986. An older reference, but dealing more specifically with cluster stars, is Freeman and Norris 1981. The basic fact which has emerged from recent work is that there is a clear indication that abundance ratios are stable within population I and within population II, with some differences between both. and a transition occuring in the intermediate population with metallicities between $1 / 10 Z_{0}$ and $1 / 3 Z_{0}$. The most well established difference between the two populations is the enhancement of oxvgen in population II versus the iron abundance bv a factor of about 3 (Clegg et a1. 1981). The case of nitrogen is very peculiar. This element does follow the abundance of iron most of the time. but is sometimes very overabundant with respect to iron in a few subdwarfs. Magnesium and silicium have moderate overabundances with respect to iron in bonula-. tion II $(0.5$ and 0.3 dex respectivelv). Heavy elements. produced bv the $s$ or the $r$ processes. either show some overdeficiency with respect to iron on follow the iron abundance. Except for nitrogen, for which a primary mechanism of production is still coniectural, these differences are explainable by recent models of galactic evolution (Greggio and Matteucci 1985, Matteucci 1986). The predicted halo composition agrees fairly well with the observed one if one assumes that the halo composition is set only by SN II explosions whereas the chemical composition of population I is set by slower processes as well (SN I, planetary nebulae ejectae). Fig. 5 and 6 illustrate this point. The success of this interpretation makes more likely that elements produced after the BigBang, found in population II, have been produced by normal SN II with 0 stars progenitors rather than in VMO's, as in Carr et al. 1984 proposal. This, however, leaves the choice between the Truran and Cameron 1971 proposal, and the self-pollution explanation.

\section{CONCLUSION}

In globulars, and more generally in population II :

i) helium and lithium have their primordial abundances

ii) element products of stellar nucleosynthesis are always present at some level, with a mean abundance of about $1 / 30^{\text {th }}$ of the solar value an a more or less log-normal distribution with $\sigma=0.3$ dex. 


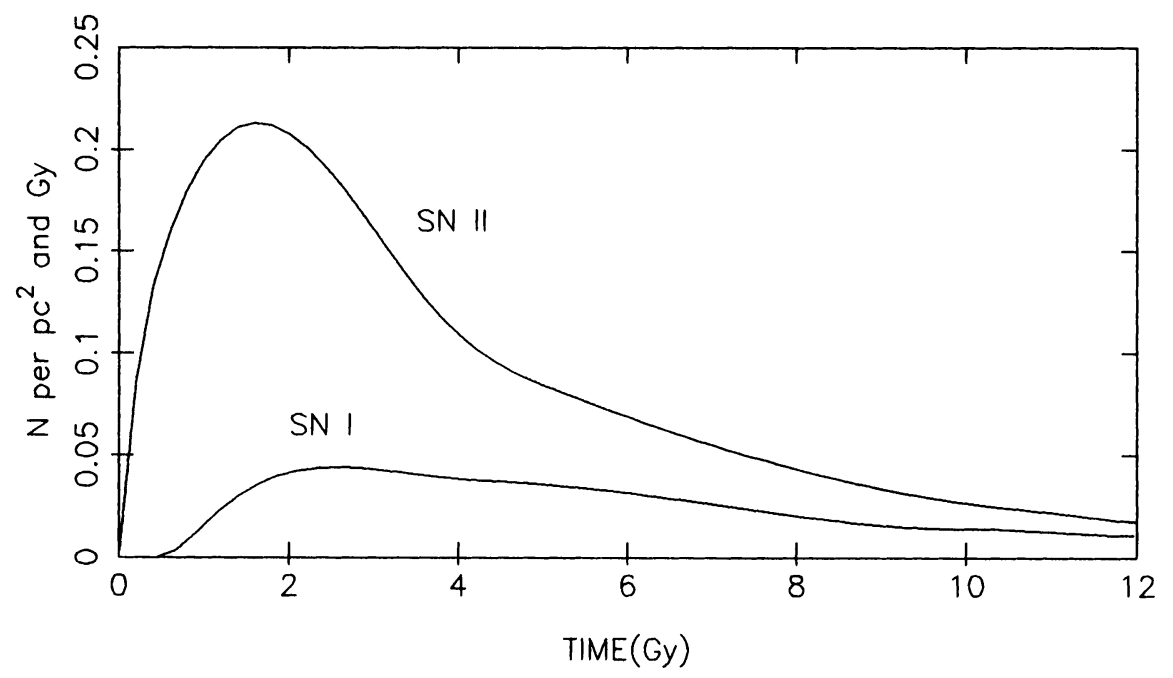

Fig. 5. Occurence of supernovae of type I and II in the life-time of the Galaxy according to Greggio and Matteucci 1985. Note that only SN II can account for the initial enrichment of the Galaxy.

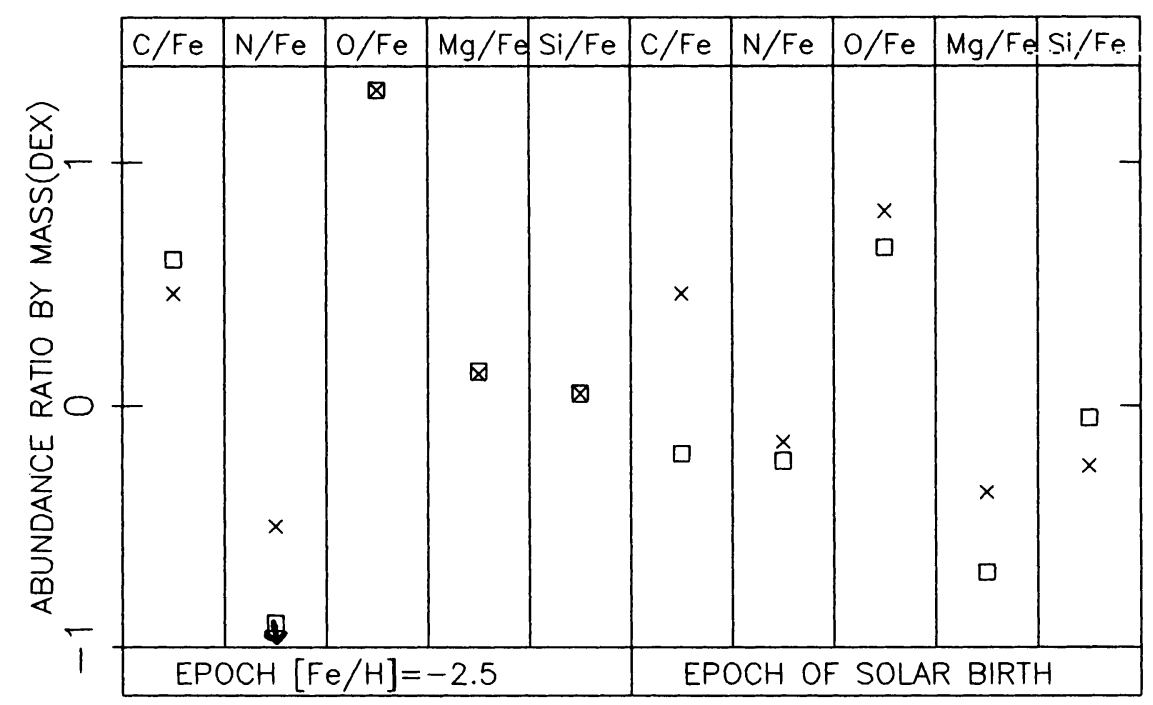

Fig. 6. Predicted ( $(\boldsymbol{a})$ and observed $(\boldsymbol{X})$ ratios of some elements i) early in the galactic life $i i)$ at the time of solar birth. Note the good fit in case i) (according to Matteucci 1986, with new ${ }^{12} \mathrm{C}(\alpha, \gamma)^{16} \mathrm{O}$ reaction rate). 
iii) Abundance ratios are slightly different from those in population $I$ and suggest strongly that the heavy elements content of population II comes exclusively from massive SN II explosions.

iv) The absence of metal free objects may either be due to a truncation of the IMF in a first stellar generation having left no visible remnants, or to the early pollution of primordial clouds by SN II in the galaxy, before they have generated the low-mass stars we see in GC and in the halo field.

\section{REFERENCES}

Audouze, J. 1986 in Workshop on Dark Matter in the Universe, Reidel, Dordrecht, p. XXX.

Boesgaard, A. M. and Steigman, G. 1985 Ann. Rev. Astron. Astrophys. 23,319 .

Bond, H. E. 1980 Astrophys. J. Supp1. 44, 517.

Buzzoni, A., Fusi Pecci, F., Buonanno, R. and Corsi, C. E. 1983 ESO Workhop on Primordial Helium P. A. Staver, D. Kunth and K. Kjar, eds., ESO, Garching, p. 231.

Caputo, F. and Castellani, V. 1983 ESO Workhop on Primordial Helium P. A. Staver, D. Kunth and K. Kjar, eds., ESO, Garching, p. 213.

Carr, B. J., Bond, J. R. and Arnett, W. D. 1984 Astrophys. J. $277,445$.

Cayre1, R. 1986 Astron. Astrophys, in press.

Clegg, R. E. S., Lambert, D. and Tomkin, J. 1981 Astrophys. J. 250,262 .

Cole, P. W., Demarque, P. and Green, E. M. 1983 ESO Workhop on Primordial Helium P. A. Staver, D. Kunth and K. Kjar, eds., ESO, Garching, p. 235.

Davidson, K. and Kinman, T. D. 1985 Astrophys. J. Supp1. 58, 321.

Eggen, 0. J., Lynden-Be11, D. and Sandage, A. R. 1962 Astrophys. J. 136,748 .

Francois, P. 1986 Astron. Astrophys. 160, 264.

Greggio, L. and Matteucci, F. 1986 Astron. Astrophys. 154, 279.

Kunth, D. 1986 Publ. Astron. Soc. Pacific in press. (Also preprint no. 143 of the Institut d'Astrophysique de Paris).

Kunth, D. and Sargent, W. L. W. 1983 Astrophys. J. 273, 81.

Luck, R. E. and Bond, H. E. 1983 Astrophys. J. Letters 271, L75.

Matteucci, F. 1986 ESO preprint no. 434. The Effect of the New $12(\alpha, \gamma)$ 16-0 Rate on the Chemical Evolution of the Solar Neighbourhood.

Palla, F. 1983 Mem Soc. Astron. Italiana 54, 235.

Peebles, P. J. E. and Dicke, R. H. 1968 Astrophys. J. 154, 891.

Peimbert, M. 1986 in Star Forming Dwarf-Galaxies and Related objects Workshop held at the I.A.P., D. Kunth, and T. X. Thuan, eds., J. Tran Thank Van. Editions Frontieres, p. 403.

Searle, L. 1977 in The Evolution of Galaxies and Stellar Populations B. M. Tinsley and R. B. Larson, eds., Yale University Observatory, New Haven, p. 219.

Spite, M., Maillard, J. P. and Spite, F. 1984 Astron. Astrophys. $141,56$. 
Spite, M. and Spite, F. 1982 Astron. Astrophys. 115, 357.

Spite, F. and Spite, M. 1986 Astron. Astrophys. 163, 140.

Truran, J. W. and Cameron, A. G. W. 1971 Astrophys. Space Sc. 14,179 .

Wagoner, R. V. 1973 Astrophys. J. 179, 343.

Yang, J., Turner, M., Steigman, G., Schramm, D. N. and Olive, K. A.

1984 Astrophys. J. 281, 493.

Zinn, R. 1985 Astrophys. J. 293, 424.

DISCUSSION

OSTRIKER: If I understand you correctly, the current lithium abundance is much higher than that in old stars. Is it possible that whatever the process which has increased $\mathrm{Li}$ also acted before the old stars were born so that the $\mathrm{Li}$ seen in these stars is not, in fact, primordial.

WALLERSTEIN: Currently lithium is reasonably well understood in terms of spallation of interstellar heavy elements by cosmic rays. If this is correct then it would not have worked when there were no heavy elements.

CARNEY: The search for and study of extremely metal-poor stars is critical to understanding our Galaxy's history (and that of others by inference). The existence or lack of such stars remains unsettled, but Beers, Preston and Shectman (1986) and Laird, Latham and I have claimed such stars exist in numbers consistent with simple models of Galactic chemical evolution.

CAYREL: It is true that the properties of extremely metal-poor stars in the field are quite important, especially because the diffuse Pop II represents 998 of the mass of the halo. However, the statistics of these very metal-poor stars still relies on very small numbers and is not well established yet.

ZINNECKER: 1) Can you explain in more detail how you solve the problem of the chemical homogeneity of globular clusters in your supernova self-enrichment scenario? 2) Is the supernova supposed to trigger the formation of the low-mass stars of the cluster? If so, you would not expect the same chemical pollution of the protostellar fragments throughout the proto-cluster cloud.

CAYREL: Concerning your first question, the level of pollution in a single burst of star formation is set by the ratio between the number of polluting supernovae and the mass of gas polluted by them. It seems reasonable to assume that these two numbers are roughly proportional, so the resulting metallicity is more or less unique. Concerning your second question, the answer is yes. Although you are right in saying that one does not expect exactly the same pollution in all protostellar fragments throughout the cloud, my answer to the first question shows that you do not expect wide variations either. But I agree with you that the most difficult point in the self-pollution mechanism is the apparent chemical homogeneity of most globular clusters. 